\title{
ALICE ITS: Operational Experience, Performance and Lessons Learned
}

\section{E. Botta* on behalf of the ALICE Collaboration}

Università di Torino e INFN - Sezione di Torino

E-mail: elena.botta@cern.ch

\begin{abstract}
ALICE (A Large Ion Collider Experiment) is a general purpose heavy-ion experiment designed for the study of strongly-interacting matter at the extreme energy densities reached in $\mathrm{Pb}-\mathrm{Pb}$ collisions at the CERN LHC, where the deconfined phase of hadronic matter known as QuarkGluon Plasma (QGP), is formed.

The innermost detector of ALICE is the Inner Tracking System. In its first release, it consists of six cylindrical layers of silicon detectors based on different technologies: two inner layers of pixel sensors (Silicon Pixel Detector), two intermediate layers of drift sensors (Silicon Drift Detector), two outer layers of strip sensors (Silicon Strip Detector). The ITS is used for the reconstruction of primary and secondary vertices, for particle tracking, for a precise determination of the impact parameter and for particle identification at low momentum.

In this report, after a brief description of the three subdetectors, the operational experience with the first ITS implementation is summarized, the status and the performance of the detector are described and the lessons learned are discussed after the end of its operation coinciding with the completion of the LHC Run2.
\end{abstract}

The 28th International Workshop on Vertex Detectors - Vertex2019

13-18 October, 2019

Lopud, Croatia

${ }^{*}$ Speaker. 


\section{Introduction}

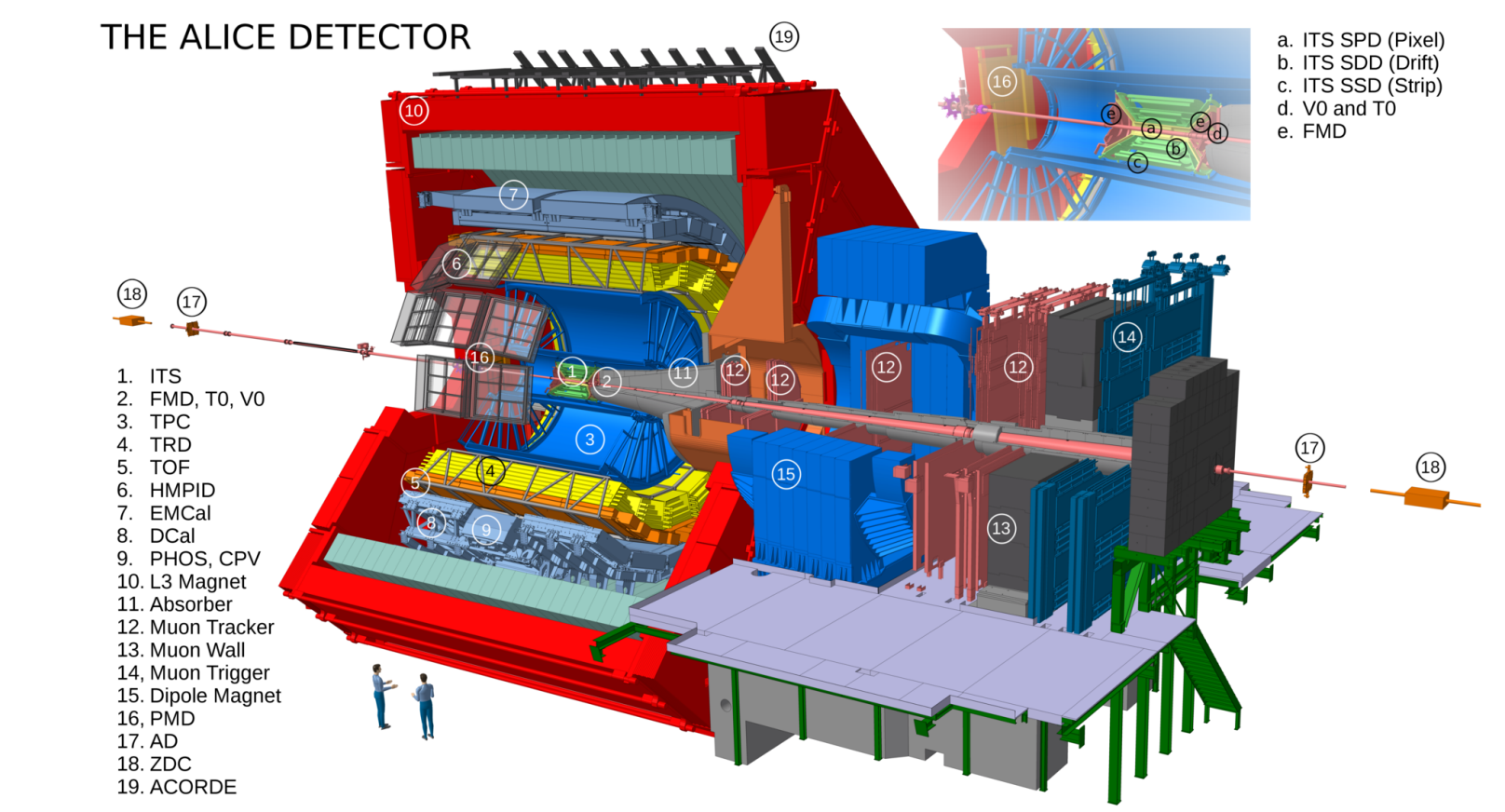

ALICE (A Large Ion Collider Experiment) [1] is designed to study the strongly-interacting matter and in particular the properties of the Quark-Gluon Plasma, a deconfined phase of matter produced in extreme energy-density conditions, such as in ultra-relativistic heavy-ion collisions. Besides $\mathrm{Pb}-\mathrm{Pb}$ collisions, the ALICE experimental program includes also $\mathrm{pp}$ and $\mathrm{p}-\mathrm{Pb}$ collisions, which are needed as reference for the study of the underlying collision dynamics.

The experimental apparatus can be divided into two different pseudorapidity regions. The forward muon arm covers the forward pseudorapidity region $-4.0<\eta<-2.5$ and is used to measure heavy-flavoured hadrons, quarkonia and light vector mesons. The central barrel covers the pseudorapidity region $|\eta|<0.9$ and the full azimuthal angle. The detectors in the central barrel are embedded in a solenoidal magnet, which provides a magnetic field up to $0.5 \mathrm{~T}$. The two main tracking detectors in the central barrel are the Inner Tracking System (ITS) [2] and the Time Projection Chamber (TPC) [3]. The low material budget of ITS and TPC $\left(\sim 10 \% X_{0}\right)$ allows the reconstruction of charged-particle trajectories in a wide transverse momentum range $(0.1-100 \mathrm{GeV} / c)$. Moreover, the central-barrel detectors allow the particle identification (PID) up to $20 \mathrm{GeV} / c$ through the measurement of the specific energy loss $\mathrm{d} E / \mathrm{d} x$ in the ITS and in the TPC and of the time of flight in the dedicated detector (TOF [4], resolution of $\sim 60 \mathrm{ps}$ in $\mathrm{Pb}-\mathrm{Pb}$ collisions).

Figure 1: Schematic of the ALICE apparatus. A zoom of the ITS is shown on the top-right corner.

\section{The Inner Tracking System (ITS)}

The ITS is the detector closest to the interaction point and has several purposes. It is used 
$\mathrm{Pb}-\mathrm{Pb}$ collisions, and the displaced vertices (secondary vertices), with a resolution better than 100 $\mu \mathrm{m}$. The precision of the primary vertex reconstruction, together with the possibility of measuring the impact parameter, i.e. the distance of closest approach of a track to the vertex, with a resolution of $\sim 60 \mu \mathrm{m}$ at $p_{\mathrm{T}}=1 \mathrm{GeV} / c$, makes it possible to study particles characterised by a short decay length, such as open heavy-flavoured hadrons. The ITS allows the reconstruction of the trajectories down to $0.1 \mathrm{GeV} / c$. Finally, the ITS can be used for PID at low transverse momentum. The ITS consists of six cylindrical layers of silicon detectors based on different technologies. A representation of the ITS within the ALICE experimental apparatus is shown in Fig. 1 and the characteristics of the ITS and its subdetectors are listed in Table 1.

\begin{tabular}{|c|c|c|c|c|c|c|c|c|}
\hline Layer & Detector & $\begin{array}{c}\text { Radius } \\
(\mathrm{cm})\end{array}$ & $\begin{array}{c}\text { Length } \\
(\mathrm{cm})\end{array}$ & Channels & \multicolumn{2}{|c|}{$\begin{array}{c}\text { Area } \\
\left(\mathrm{m}^{2}\right)\end{array}$} & $\begin{array}{l}\text { Res. }(\mu \mathrm{m}) \\
\mathrm{z} \phi\end{array}$ & $\begin{array}{c}\text { M.B. } \\
\left(\% \mathrm{X}_{0}\right)\end{array}$ \\
\hline 1 & $\mathrm{SPD}$ & 3.9 & 28.2 & $3.3 \mathrm{M}$ & 0.07 & 12 & 100 & 1.14 \\
2 & & 7.6 & 28.2 & $6.5 \mathrm{M}$ & 0.14 & & & 1.14 \\
\hline 3 & $\mathrm{SDD}$ & 15.0 & 44.4 & $43 \mathrm{k}$ & 0.42 & 35 & 25 & 1.13 \\
4 & & 23.9 & 59.4 & $90 \mathrm{k}$ & 0.89 & & & 1.26 \\
\hline 5 & $\mathrm{SSD}$ & 38.0 & 86.2 & $1.1 \mathrm{M}$ & 2.20 & 20 & 830 & 0.83 \\
6 & & 43.0 & 97.8 & $1.5 \mathrm{M}$ & 2.80 & & & 0.83 \\
\hline
\end{tabular}

Table 1: Characteristics of the ITS, layer by layer. Res. is the resolution, along the bending direction $(r \phi)$ and the beam axis $(z) . M . B$. is the material budget, expressed in terms of radiation lengths $\left(X_{0}\right)$.

\subsection{The Silicon Pixel Detector (SPD)}

The two innermost layers constitute the Silicon Pixel Detector (SPD), based on hybrid pixel detectors with binary output. The SPD consists of 120 Half-Stave modules, grouped in two HalfBarrels divided in the $z$ direction. Each Half-Barrel contains 10 Half-Sectors, each constructed from six Half-Staves, two from the first and four from the second layer. The Half-Stave is composed of two ladders, each containing one two-dimensional $200 \mu \mathrm{m}$ thick silicon sensor matrix and five $150 \mu \mathrm{m}$ thick bump-bonded readout chips each. The matrix has $256 \times 160$ reversely biased $(50 \mathrm{~V})$ $\mathrm{p}^{+} \mathrm{n}$ diodes, forming cells $50 \mu \mathrm{m}$ wide $(r \phi)$ and $425 \mu \mathrm{m}$ long $(z)$. The binary output is readout at $10 \mathrm{MHz}$. The detector is equipped with a $\mathrm{C}_{4} \mathrm{~F}_{10}$ evaporative cooling system.

The SPD is the only ITS subdetector which contributes to the ALICE trigger (L0). Each pixel readout chip outputs the data of the fired pixels and one Fast-OR signal set to 1 if at least one of the 8192 related pixels is fired. The Fast-OR signals are produced at $10 \mathrm{MHz}$ and are used by the ALICE Central Trigger Processor to produce High Multiplicity L0 trigger, based on the number of SPD tracklets (segments obtained coupling pairs of points on the two layers), and Double-Gap Diffractive trigger, in which two cones are looked for in mid-rapidity region and selections are applied to the opening angle as well as to the number of tracklets. 


\subsection{The Silicon Drift Detector (SDD)}

The Silicon Drift Detector (SDD) equips the two intermediate layers of the ITS. It consists of 260 drift modules, each composed of a $300 \mu \mathrm{m}$ thick drift sensor and two custom hybrid readout boards. The sensitive area of the module is divided into two drift regions along the bending direction $(r \phi)$ by the central cathode at $-1.8 \mathrm{kV}$. In each drift region and on both detector surfaces, 291 $\mathrm{p}^{+}$cathode strips $(120 \mu \mathrm{m}$ pitch) fully deplete the detector and generate a uniform electric drift field $(\sim 500 \mathrm{~V} / \mathrm{cm})$, parallel to the module surface, towards 256 collection anodes ( $294 \mu \mathrm{m}$ pitch) aligned to the beam axis.

The drift speed is about $6.7 \mu \mathrm{m} / \mathrm{s}$ and is monitored by means of MOS charge injectors: in each drift region there are three lines of 22 MOS injectors, close to the anodes, in the middle of the drift region and close to the central cathode. The drift speed is measured in specific calibration runs at the beginning of each LHC fill. The analog SDD information is digitalized by 10-bit ADCs at 20 $\mathrm{MHz}$ at the level of the front-end electronics developed on CMOS $0.25 \mu \mathrm{m}$. The SDD is provided with a leak-less water cooling system.

\subsection{The Silicon Strip Detector (SSD)}

The two outermost layers of the ITS constitute the Silicon Strip Detector (SSD). It consists of 1698 modules, each composed of 768 double-sided strip sensors, with a thickness of $300 \mu \mathrm{m}$, connected with two HAL25 front-end chips. The analog signals are extracted from the detector and digitalized by 12-bit ADCs directly in the readout electronics placed outside the ALICE solenoid magnet.

The strips on the P-side and on the N-side of the sensor have a pitch of $95 \mu \mathrm{m}$, a length of 40 $\mathrm{mm}$ and angles of, respectively, $7.5 \mathrm{mrad}$ and $27.5 \mathrm{mrad}$ with respect to the beam axis, to define a stereo angle of $35 \mathrm{mrad}$, which is a compromise between stereo view and reduction of ambiguities due to high particle density, to ensure the best position resolution in the bending direction. The $\mathrm{p}^{+} \mathrm{n}$ reverse bias is optimized in the range $20-80 \mathrm{~V}$. The SSD is provided with a leak-less water cooling system shared with the SDD and, due to the sensitivity of the detector to the air humidity, an air dryer system is also used to keep the absolute humidity between 1 and $1.5 \mathrm{~g} / \mathrm{kg}$.

\section{The ITS operational performance}

The ITS operational efficiency is summarized in Table 2, which reports the availability of the ITS subdetectors, expressed as the fraction of the total ALICE data-acquisition time each subdetector participated in, and the acceptance, indicated as the fraction of modules in acquisition. Generally, for all the detectors the availability was stable during Run2 and improved with respect to Run1. The improvement was possible thanks to the firmware update of the readout electronics, to the automation of recovery procedures and to the interventions that took place during Run 1 and the following Long Shutdown (LS1).

In this regard, it is useful to remember that in 2011 only $63 \%$ of the SPD could be powered on, due to an insufficient flow of the liquid freon in the cooling system, with filters clogged by metal and graphite fragments. Clogged filters have been drilled in 2012-2013, restoring the number of active modules to $92 \%$ at the end of Run1. During Run2, the SPD acceptance and availability were 


\begin{tabular}{|c|ccc|ccc|}
\hline \multirow{2}{*}{} & \multicolumn{3}{|c|}{ Availability (\%) } & \multicolumn{3}{c|}{ Acceptance (\%) } \\
\cline { 2 - 7 } & SPD & SDD & SSD & SPD & SDD & SSD \\
\hline Run1 (2013) & 96.0 & 92.0 & 96 & 92 & 87 & 91 \\
\hline Run2 (2015-2016) & 99.6 & 98.4 & 98.6 & 93 & 83 & 91 \\
Run2 (2017) & 99.9 & 99.5 & 100.0 & 93 & 82 & 91 \\
Run2 (pp 2018) & 99.6 & 99.8 & 99.8 & 92 & 81 & 91 \\
Run2 (Pb-Pb 2018) & 98.8 & 95.7 & 99.6 & 92 & 81 & 91 \\
\hline
\end{tabular}

Table 2: Availability and acceptance of the three ITS subdetectors during the last part of Run1 and Run2, indicated as fraction of data acquisition-time and of number of active modules, respectively. In the 2018 $\mathrm{Pb}-\mathrm{Pb}$ data taking SDD show a reduced availability due to SDD exclusion from rare triggers.

very stable: no radiation effect appeared as increase in noisy channels and temperature thanks to the reduced luminosity leveled to $2.6 \mathrm{~Hz} / \mu$ barn in the ALICE interaction point that produced a low total ionizing dose (TID) at the SPD position (17.4 krad). Still, a continuous slowly increasing trend of the bulk current was observed for some Half Staves of the innermost layer, and was fixed by a careful tuning of current limits before each data taking. Additionally, another effect was

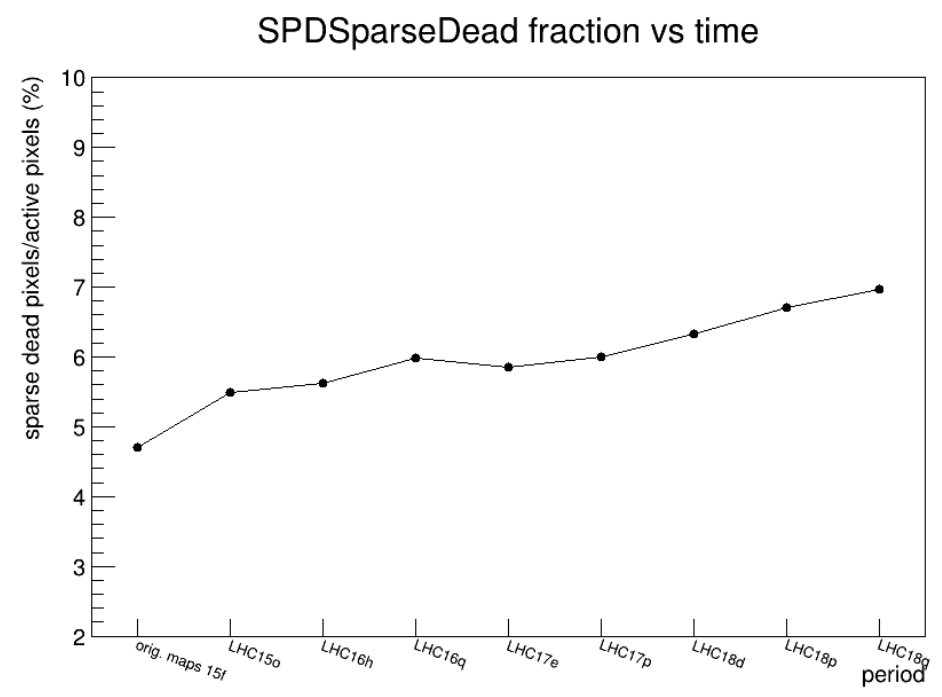

Figure 2: SPD sparse dead pixel fraction trend in LHC Run2 beam periods from 2015 to 2018.

observed starting from the 2015 data taking: a continuous slight increase of the fraction of sparse dead pixels (from $\sim 4.6 \%$ in 2015 to $\sim 7 \%$ in 2018, see Fig. 2). The dead pixels are mainly located at the edges of the bump-bonded readout chips: this is consistent with a bump-bonding detachment process probably induced by mechanical and thermal stress. This kind of issue is overcome in the new release of the ITS that is based on monolithic active pixel sensors (MAPS) [5]. Globally, no effect on the performance in terms of detection efficiency and space accuracy has been observed in Run2 for SPD. 
Concerning the SDD, the most recurring problems during Run1 were the loss of communication of one module and the presence of an error in the Common Data Header (CDH), interpreted as a consequence of a Single Event Upset (SEU). The recovery time to reset the configuration of the front-end electronics was reduced to $800 \mu$ s in the transition between Run1 and Run2, by upgrading the readout boards with more performing FPGA; thanks to these new boards, a new firmware was also designed, with an automatic procedure to detect event by event noisy chips and eventually to reload the configuration parameters without stopping the data acquisition. This improved the availability of the detector and reduced the global acquisition dead time. On the other hand, since the beginning the fraction of active modules was lower with respect to the other subdetectors, as indicated in Table 2, because of communication loss with some modules during installation and Run1. Also, during an LHC beam dump close to ALICE, the inner SDD layer suffered from high radiation exposure causing the loss of many MOS injectors. During all Run2 SDD behaved well, featuring stable fraction of active modules, stable and low noise level ( $\sim 350$ equivalent electrons), very stable baseline and gain.

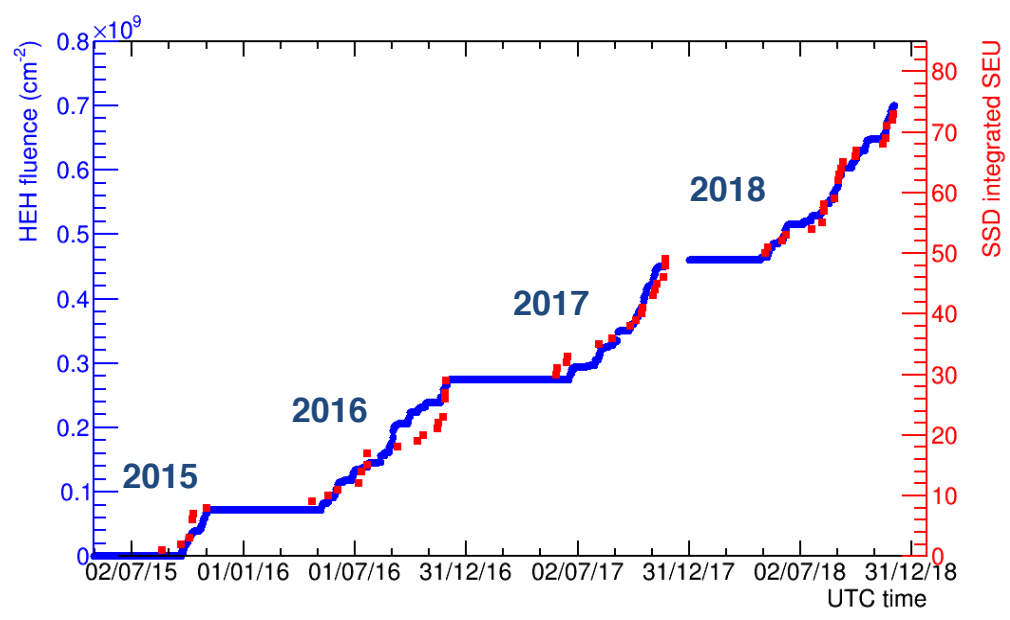

Figure 3: Integrated number of SEU events and integrated HEH fluence in Run2 (2015-2018).

One of the most recurrent problems with the SSD in Run1 and Run2 was the occurrence of SEU events affecting the SRAM FPGA of the Front End Read Out Modules (FEROM). FEROM modules are located in the cavern, close to the solenoid magnet, in a region where the TID at the end of Run2 was $\sim 0.34 \mathrm{krad}$ and SEUs were not expected. In Fig. 3 the integrated number of SEUs since 2015 is shown, together with the integrated fluence of High Energy Hadrons (HEH), measured with a sensor within the FEROM crate. The correlation between the number of SEU events and the integrated fluence is evident and indicates that the origin of these events is actually due to the beam presence and not to electronics ageing effects. After Run1 some countermeasures were implemented. In particular, the PROMs in the FEROMs were replaced with new radiation tolerant PROMs and the FPGA firmware was updated, reducing the recovery time from 20 minutes to 5 minutes in Run2. At the beginning of Run2, SSD suffered also from air humidity leading to an increase of leakage currents: interventions on the ventilation machine in 2015 and the addition of an air dryer system in 2016 allowed to fix these issues. In the $2018 \mathrm{~Pb}-\mathrm{Pb}$ data taking SSD was the most stable of the ITS detectors. 


\section{The ITS detector and physics performance}

The ITS plays a crucial role in the reconstruction of the primary and secondary vertices. The algorithm used to perform this task is based on an iterative procedure using tracks reconstructed in the whole ALICE central barrel with the ITS and the TPC. To extend the transverse momentum acceptance down to $0.1 \mathrm{GeV} / c$ and to recover tracks not reconstructed in TPC, an ITS-standalone tracking algorithm has been developed. The efficiency for tracks with at least 2 points in ITS is $\geq 95 \%$ and is constant in the transverse momentum range; Fig. 4 (left) shows the efficiency for tracks with (at least) one hit in SPD and a hit in TOF for $2016 \mathrm{p}-\mathrm{Pb}$ collisions at $\sqrt{s_{\mathrm{NN}}}=5.02 \mathrm{TeV}$.

As anticipated, the ITS contributes to the ALICE particle identification measuring the ionizing energy loss $\mathrm{d} E / \mathrm{d} x$ in the four outermost layers via the readout of the analog signal proportional to the charge released in the detector layers [6]. Fig. 4 (right) shows an example of the mean energy deposition as a function of the momentum measured in $\mathrm{Pb}-\mathrm{Pb}$ collisions at $\sqrt{s_{\mathrm{NN}}}=5.02 \mathrm{TeV}$. The ITS particle identification combined with the standalone tracking allows for a $K-\pi$ and a $K-p$ separation in the range $(0.1-0.45) \mathrm{GeV} / c$ and $(0.1-1) \mathrm{GeV} / c$ respectively.
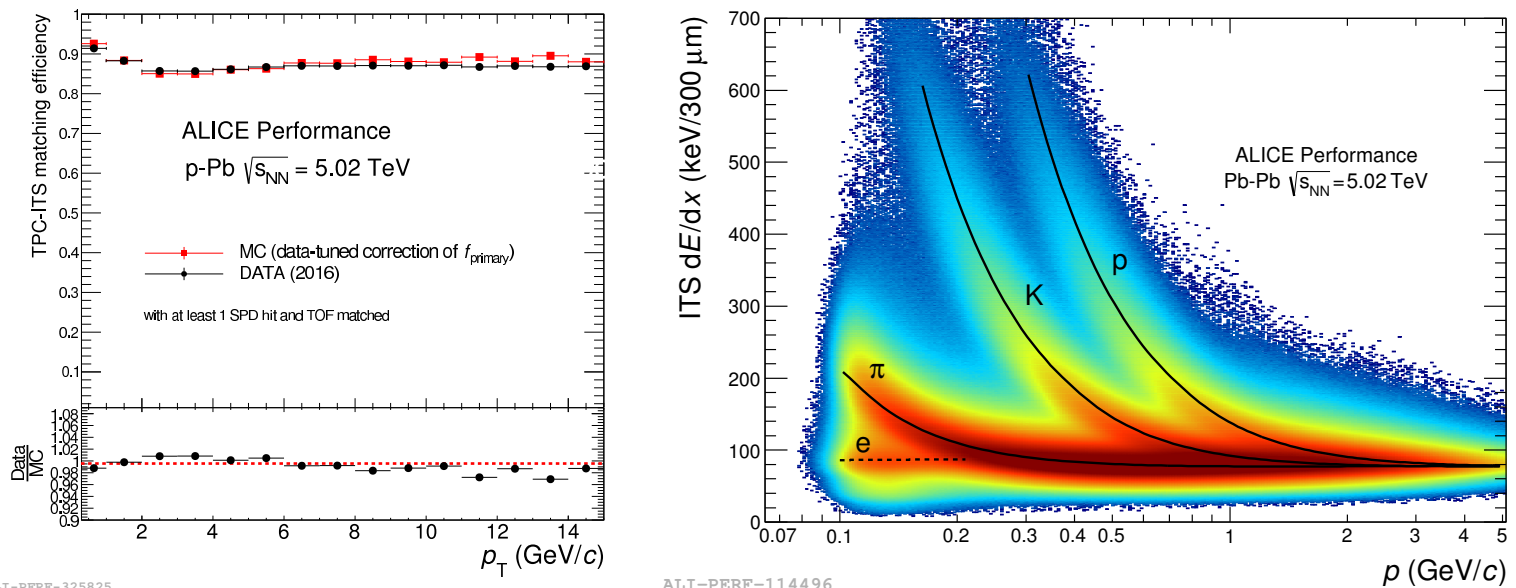

Figure 4: Run2 ITS detector performance. Left: TPC-ITS matching efficiency vs momentum for tracks with at least 1 hit in SPD and a hit in TOF in $\mathrm{p}-\mathrm{Pb}$ collisions at $\sqrt{s_{\mathrm{NN}}}=5.02 \mathrm{TeV}$. Right: ITS specific energy loss of electrons, pions, kaons and protons vs momentum in $\mathrm{Pb}-\mathrm{Pb}$ collisions at $\sqrt{s_{\mathrm{NN}}}=5.02 \mathrm{TeV}$.

The resolution of the track impact parameter $d_{0}$, calculated as the distance of closest approach of a given track to the vertex, allows to define the tracking precision of the system. In Fig. 5 (left) the impact parameter resolution in the $x-y$ plane, for identified ITS-TPC tracks, is reported as a function of the transverse momentum for three different collision systems [6]. For all of them the $d_{0}$ resolution is about $60 \mu \mathrm{m}$ at $1 \mathrm{GeV} / c$; the contribution of the primary vertex resolution is not subtracted. The precise measurement of the track impact parameter and the capability to separate the primary and secondary vertex allows the inclusive reconstruction of rare particles via their hadronic decays: Fig. 5 (right) shows the invariant mass spectrum of the short-lived $(c \tau \sim$ $123 \mu \mathrm{m}) D^{0}$ meson decaying to $K \pi$ pairs.

\section{Lessons learned and Conclusions}

The ALICE Inner Tracking System has been successfully in operation during LHC Run1 and 

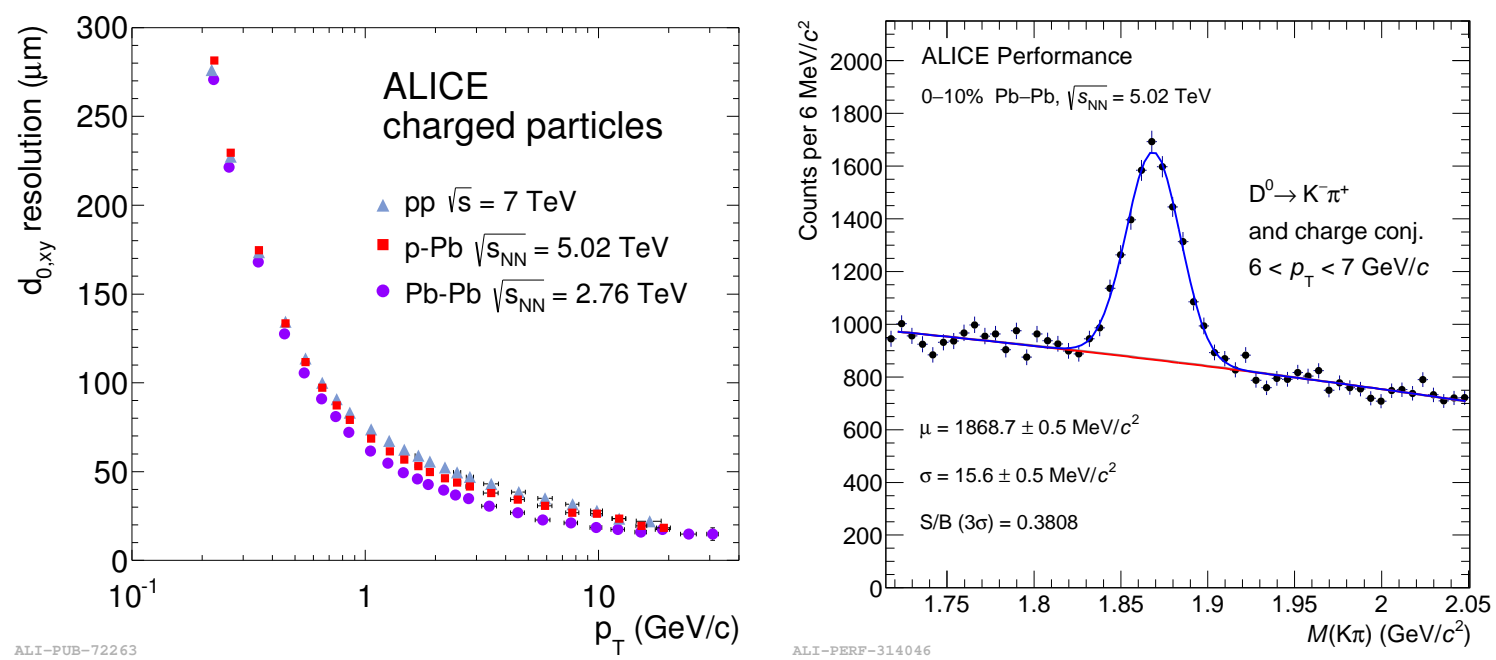

Figure 5: Run2 ITS physics performance. Left: impact parameter resolution in the $x-y$ plane as a function of the transverse momentum in pp collisions at $\sqrt{s}=7 \mathrm{TeV}, \mathrm{p}-\mathrm{Pb}$ collisions at $\sqrt{s_{N N}}=5.02 \mathrm{TeV}$ and in $\mathrm{Pb}-$ $\mathrm{Pb}$ collisions at $\sqrt{s_{N N}}=2.76 \mathrm{TeV}$. Right: $K \pi$ invariant mass in $D^{0}$ mass region for central $\mathrm{Pb}-\mathrm{Pb}$ collisions at $\sqrt{s_{N N}}=5.02 \mathrm{TeV}$.

Run2, with high efficiency and stable participation in $\mathrm{pp}, \mathrm{p}-\mathrm{Pb}$ and $\mathrm{Pb}-\mathrm{Pb}$ data takings. During Run2, procedures have been implemented to minimize the recovery time related to SEU events and to issues arising when reloading the configuration parameters of the readout electronics. SPD fully contributed to the L0 trigger algorithms. SPD, SDD and SSD performed remarkably well and according to the specifications, the physics performance was in agreement with the design requirements and was stable in time.

At the end of operation of the first release of the ITS some lessons learned can be discussed. As a global consideration, the three subdetectors, based on different technologies, actually worked as three independent detectors with different features, needs and peculiarities, so that no standardization was possible in procedures related to front-end electronics configuration, data acquisition, detector calibration. Indeed, each subdetector had its own Detector Control System (DCS) to remotely control the underground hardware, to apply specific operation strategies (e.g. SEU recovery and security operations during beam injections), to monitor operational conditions and data quality for spotting misconfigurations during data taking (Data Quality Monitor, DQM). In the same way, each subdetector had its own Experiment Control System (ECS) to perform specific operations like stand alone runs and calibrations; also the offline Quality Assurance procedure (QA) was different.

For SPD issues have appeared in the operation of the cooling system that, in future implementations, could be eliminated by an even more attentive choice of the position of the filters on the feeding lines. A second point to be taken into account are the residual effects of mechanical and thermal stress actions developed during the detector running on the sensors bump bonded to the readout chips, which produced an (almost negligible) increase of the dead pixels number at the chip edges. Both aspects have been fully overcome in the Upgrade release of the ITS [5], presently in post assembly test phase: it is composed of seven layers of MAPS (no bump bonding!) and the upgraded Inner Barrel features a power absorption about 1/10 than the SPD one. 
For SDD the main lesson learned is related to the drift speed calibration. It must be underlined that ALICE could operate successfully an SDD system exploiting at best the two dimensional capabilities provided by this technology thanks to several solutions implemented in the hardware and in the readout and to a complex calibration scheme. The resolution along the anode rows (see Table 1) was obtained by the well established collected charge centroid technique while the resolution in the drift direction relied on the determination of the drift velocity that is sensitive to temperature gradients in the SDD volume and to temperature variations with time. Calibration runs were done at the beginning of each LHC fill, based on the signals of the three MOS injector lines implanted at known distances from the collection anodes. The drift field, however, was intrinsically not uniform enough to allow, this way, an evaluation of the hit position along the drift direction at the precision level needed to fulfill the design requirements; to obtain the final resolution a correction for the non-uniformity of the drift field was calibrated for each LHC fill based on the precise information of the neighboring ITS layers.

For SSD the main lesson is related to humidity control of the air surrounding the detector, to avoid increasing leakage currents that can lead to permanent damages. The second point, actually the first one in terms of frequency, is related to SEUs occurrence, which can only be fixed either by moving the frontend electronics farer from the beam line or by adding shielding material in front of it. This was not viable in the past ALICE setup and has been attentively considered for the Upgraded ITS.

\section{References}

[1] "The ALICE experiment at CERN LHC" $\mathrm{K}$. Aamodt et al. [ALICE Collaboration] JINST 3 (2008) S08002.

[2] "ALICE technical design report of the inner tracking system (ITS)" G. Dellacasa et al. [ALICE Collaboration] CERN-LHCC-99-12

[3] "ALICE technical design report of the time projection chamber (TPC)" G. Dellacasa et al. [ALICE Collaboration] CERN-LHCC-2000-001

[4] "ALICE technical design report of the time-of-flight system (TOF)" G. Dellacasa et al. [ALICE Collaboration]. CERN-LHCC-2000-012

[5] "Technical Design Report for the Upgrade of the ALICE Inner Tracking System" B. Abelev et al. [ALICE Collaboration] J. Phys. G 41, 087002 (2014). CERN-LHCC-2013-024, ALICE-TDR-017

[6] "Performance of the ALICE experiment at the CERN LHC" B. Abelev et al. [ALICE Collaboration] Int. J. Mod. Phys. A 29, 1430044 (2014). CERN-PH-EP-2014-031 